\title{
Engineering of Commercial Polyamide Microfiltration Membrane via Robustly Immobilizing Gallic Acid-Modified Silver Nanoparticles for the Removal of Antibiotics and Antibiotic-Resistant Bacteria
}

Ruifeng Liang, Ruiquan Yu ${ }^{\mathrm{b}}$, Rui Wang ${ }^{\mathrm{b}}$, Zhixuan Zhou ${ }^{\mathrm{c}}$, Gongyan Liu ${ }^{\mathrm{b}, *}$

${ }^{a}$ The State Key Laboratory of Hydraulic and Mountain River Engineering, Sichuan

University, Chengdu 610065, China

${ }^{\mathrm{b}}$ The Key Laboratory of Leather Chemistry and Engineering of Ministry of Education,

National Engineering Research Center of Clean Technology in Leather Industry,

College of Biomass Science and Engineering, Sichuan University, Chengdu 610065,

China

'Max Planck Institute for Polymer Research, Ackermannweg 10, Mainz 55128, Germany

*Corresponding Author, E-mail: lgy3506@scu.edu.cn 


\section{MATERIALS AND METHODS}

\section{Materials}

Disodium hydrogen phosphate dihydrate $\left(\mathrm{Na}_{2} \mathrm{HPO}_{4} \cdot \mathrm{H}_{2} \mathrm{O}\right)$ was purchased from Shanghai Macklin Biochemical Co., Ltd. (Shanghai, China). Silver standard solution $\left(1000 \mu \mathrm{g} \cdot \mathrm{mL}^{-1}\right)$ was purchased from Zhongbiao Technology Co., Ltd. (Jinan, China). Ethanol and glutaraldehyde 50\% were purchased from Kelong chemistry company (Chengdu, China). LIVE/DEAD Baclight ${ }^{\mathrm{TM}}$ bacterial viability kit (L-7007, Invitrogen) was purchased from Thermo Fisher Scientific (China) Co., Ltd. (Shanghai, China).

\section{Characterization}

The morphological characterization of GA@AgNPs was performed by transmission electron microscope (ZEISS Libra 200 FE). The hydrodynamic diameters of GA@AgNPs were measured using a Zeta-sizer Nano ZS (Malvern, UK). UV-Vis absorption spectra of GA@AgNPs were recorded with a U-3900H Spectrophotometer (HITACHI, Japan). UV-Vis absorption spectra of GA@AgNPs-PMF were recorded with a UV-3600 UV-VIS-NIR Spectrophotometer (SHIMADZU, Japan). The surface morphologies of the prepared GA@AgNPs-PMF samples were accomplished using FESEM (JEOL, Japan) operated at an accelerating voltage of $15 \mathrm{kV}$. The elemental composition and changes of the virgin PMF and GA@AgNPs-PMF membranes were characterized by X-ray photoelectron spectroscopy (XPS, XSAM800, Kratos Analytical 
Ltd., UK), and EDX spectrometer coupled with the FESEM. The surface zeta-potential value of GA@AgNPs-PMF was detected using the streaming potential method via an electrokinetic analyzer (Mütek SZP-10, BTG, Germany) with $1 \mathrm{mmol} / \mathrm{L} \mathrm{KCl}$ solution electrolyte solution. Water contact angle (WCA) was measured by a DSA30 contact angle goniometer (Krüss GmbH, Germany), dropping $2.0 \mu \mathrm{L}$ of water onto the surface of super-hydrophilicity GA@AgNPs-PMF. The WCA was obtained from the average values of three measurements per sample. ATR-FTIR (Thermo Fisher Nicolet Is5) was used to observe the surface chemical structure. The reactive radicals were detected using electron paramagnetic resonance (EPR, Bruker E500-10/12).

\section{The Silver Loading Content and Stability of GA@AgNPs-PMF}

Briefly, 100 mg of dry GA@AgNPs-PMF sample was nitrated by $5 \mathrm{~mL}$ of $\mathrm{HNO}_{3} / \mathrm{H}_{2} \mathrm{O}_{2}$ mixture solution $(7: 3, \mathrm{v}: \mathrm{v})$. Then the solution was diluted by DI water to $100 \mathrm{~mL}$ for ICP-AES analysis to determine the silver loading content. The silver loading stability of GA@AgNPs-PMF sample was determined by measuring the leaching amount of silver under static and dynamic conditions, respectively. For testing stability under static condition, 50 mg GA@AgNPs-PMF sample was immersed into 50 mL DI water with continuous shaking $(150 \mathrm{rpm})$ at room temperature and the water was replaced with $50 \mathrm{~mL}$ fresh DI water every $24 \mathrm{~h}$. The silver content in DI water was measured by ICP-AES to determine the leaching of silver from GA@AgNPs-PMF sample. After 7 days, the changes of chemical element contents of GA@AgNPs-PMF 
were analyzed by XPS instrument (XPS, XSAM800, Kratos Analytical Ltd., UK).

For evaluating the dynamic filtration stability, $700 \mathrm{~mL}$ DI water was filtered through circular GA@AgNPs-PMF sample by gravity-driven. During the gravity-driven filtration, the depth of the DI water above GA@AgNPs-PMF sample was maintained at $10 \mathrm{~cm}$ to provide constant hydraulic pressure, and the filtrate was collected and the silver concentration was measure by ICP-AES.

\section{Antibacterial Activities of GA@AgNPs-PMF}

Briefly, $0.1 \mathrm{~g}$ dry membrane sample was cut into pieces and put into $20 \mathrm{~mL}$ of PBS solution with bacterial concentration of $10^{6} \mathrm{CFU} \cdot \mathrm{mL}^{-1}$ in an Erlenmeyer flask, incubating at $37^{\circ} \mathrm{C}$ with shaking at $150 \mathrm{rpm}$ on an rotary shaker. At $0 \mathrm{~min}$ and $10 \mathrm{~min}$, $20 \mu \mathrm{L}$ bacterial solution was withdrawn from flask and spread on NA petri plate, with incubating at $37^{\circ} \mathrm{C}$ overnight. The colony-forming units were then counted:

$\log$ reduction $=\log \left(\mathrm{CFU}_{0 \min } \mathrm{mL}^{-1}\right)-\log \left(\mathrm{CFU}_{10 \min } \mathrm{mL}^{-1}\right)$

$\%$ Kill $=\left(\mathrm{CFU}_{0 \min } \mathrm{mL}^{-1}-\mathrm{CFU}_{10 \min } \mathrm{mL}^{-1}\right) / \mathrm{CFU}_{0 \min } \mathrm{mL}^{-1} \times 100 \%$

For investigating the repeatedly bactericidal activities of membrane samples, the above tested membranes were taken out from bacterial solution after $10 \mathrm{~min}$ and washed by 20 $\mathrm{mL}$ PBS for $10 \mathrm{~min}$. Then, the killing efficiency was tested again by using the same procedure as described above and defined as one repeated challenge. In this study, the three repeated challenges were tested. 


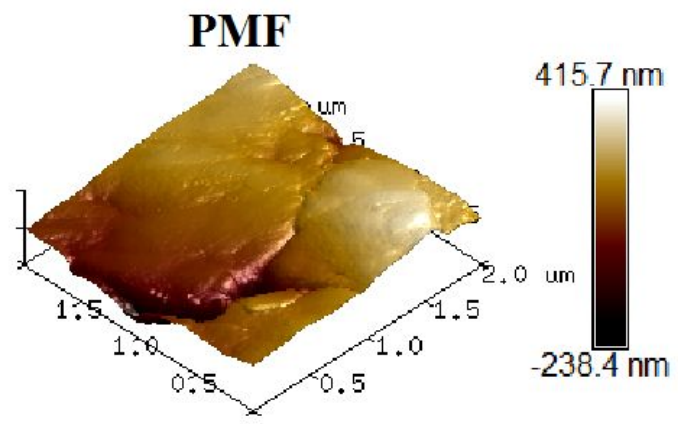

Height Sensor

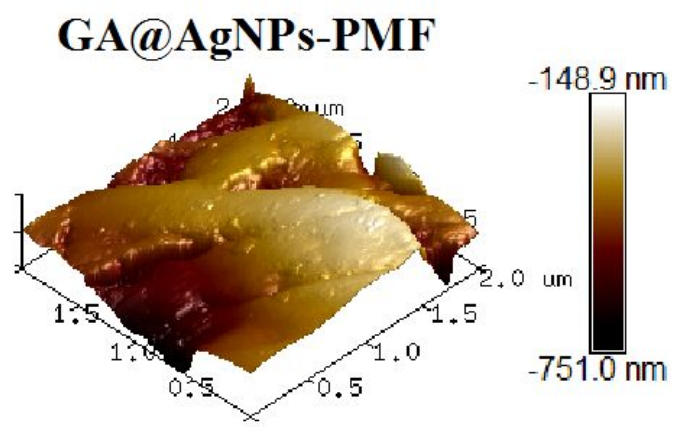

Height Sensor

Figure S1. AFM images of the virgin PMF and GA@AgNPs-PMF membranes.
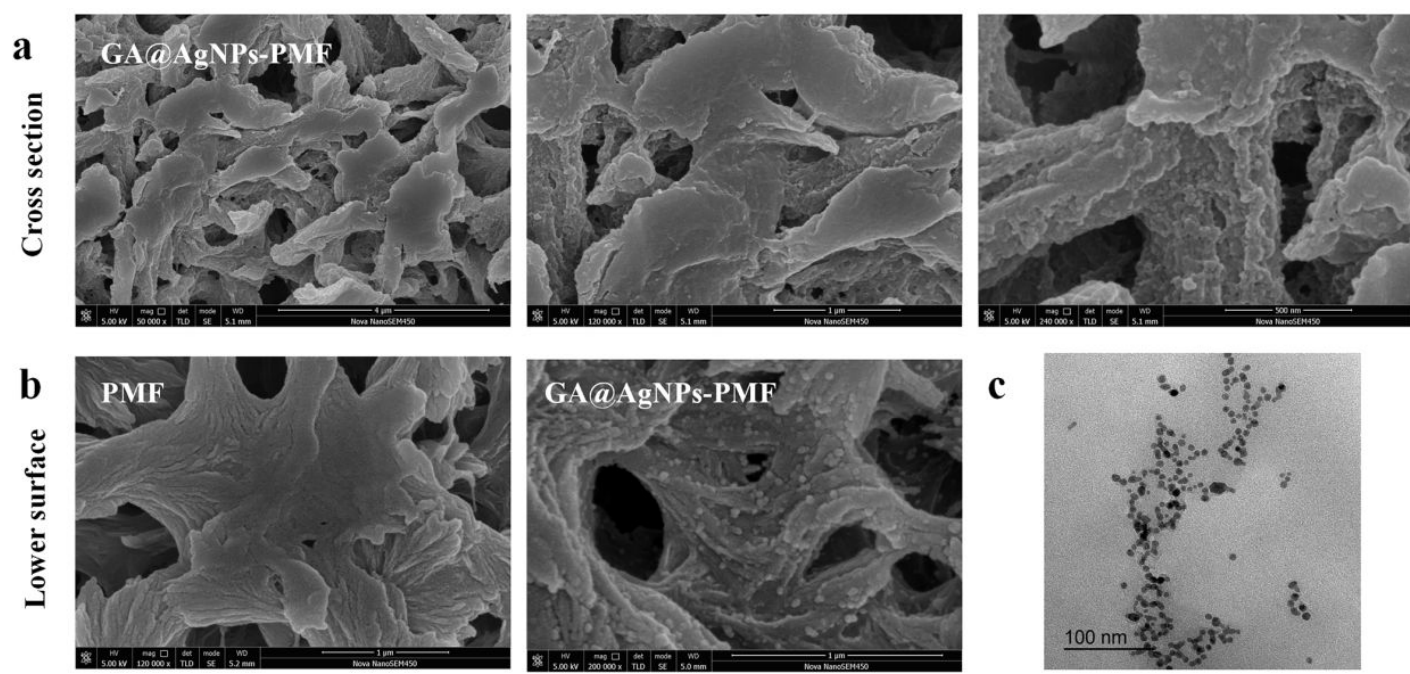

d
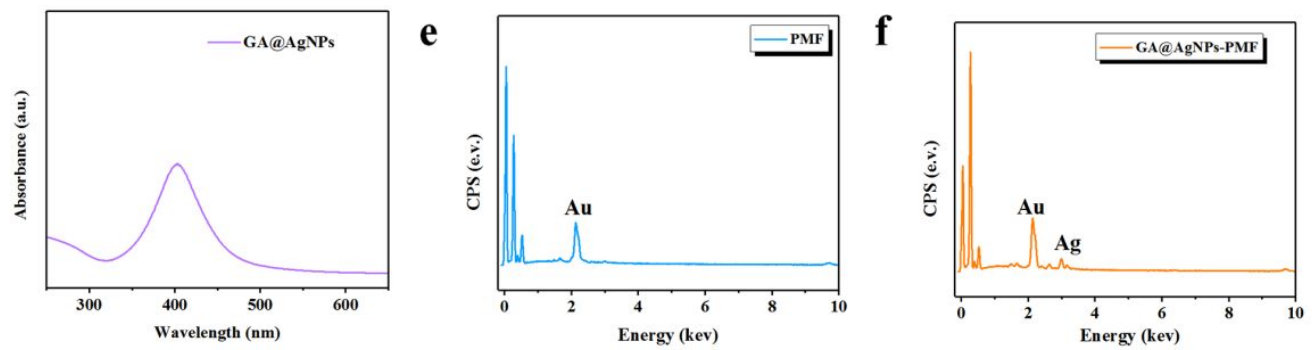
Figure S2. (a) FESEM images of cross section morphologies with different magnifications of GA@AgNPs-PMF membranes. (b) FESEM images of lower surface of the PMF and GA@AgNPs-PMF membranes. (c) TEM images and (d) UV-vis spectroscopy of AgNPs leaching from GA@AgNPs-PMF membranes after ultrasonic treatment. EDS spectrum of the (e) PMF and (f) GA@AgNPs-PMF membranes.

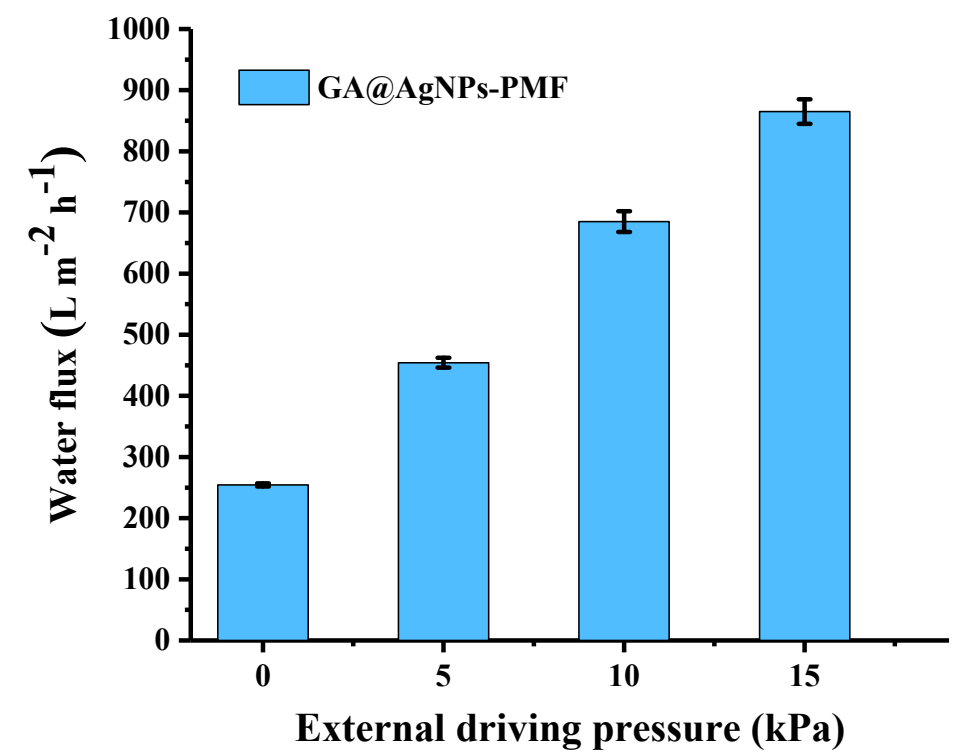

Figure S3. (a) Permeation fluxes of GA@AgNPs-PMF membrane at different external driving pressures. 